\title{
Hodgkin's lymphoma is a rare form of clonal haematological non-mast cell disease in systemic mastocytosis
}

\author{
Gorana Gasljevic ${ }^{1}$, Biljana Grcar-Kuzmanov ${ }^{1}$, Alenka Grosel², Matjaz Sever ${ }^{3}$ Barbara Gazic ${ }^{1}$ \\ and Veronika Kloboves-Prevodnik ${ }^{4^{*}}$
}

\begin{abstract}
Background: The association of systemic mastocytosis (SM) with a non-mast cell haematological neoplasm represents a specific subtype of mastocytosis termed systemic mastocytosis with associated haematological non-mast cell disease (SM-AHNMD). The overwhelming majority of the associated neoplasms are of myeloid origin, while lymphoid neoplasms associated with SM have been reported rarely. Association of SM with Hodgkin's lymphoma (HL) is exceedingly rare; so far, only two cases of HL as associated hematological non-mast cell disease in systemic mastocytosis have been published in the recent English literature.

Case: We present a case of a 37-year-old otherwise healthy male who was referred to our institution because of a one-month lasting dysphagia of both hard and liquid food. Physical examination showed tumour in the left jugular area measuring $2 \mathrm{~cm}$ in the largest diameter while computer tomography of the thorax revealed a $5.2 \mathrm{~cm}$ large, hypodense, soft tissue tumour between the trachea and left arteria carotis communis. On the basis of FNAB findings, the diagnosis of a "neutrophil-rich" Hodgkin's lymphoma was established. Excisional biopsy of mediastinal tumor showed lymphoid neoplasm with morphology and immunophenotype consistent with nodular sclerosis classical Hodgkin's lymphoma (NSCHL). Bone marrow trephine biopsy and the MGG-stained smear of the bone marrow aspirate performed for lymphoma staging revealed an existence of systemic mastocytosis which was unexpected and incidental finding. Mast cells were highlighted by CD117 and tryptase immunostainings while CD25 positivity of mast cells was consistent with their neoplastic phenotype.There were no $\mathrm{HL}$ infiltrates present in the bone marrow.
\end{abstract}

Conclusion: We report a very rare combination of systemic mastocytosis with Hodgkin's lymphoma as associated clonal haematological non-mast cell lineage disease. Systemic mastocytosis was an unexpected finding. The diagnosis of SM in bone marrow in our case was straight-forward, but it can be difficult in the case of reactive lymphoid aggregates or a difficult distinction between SM and HL infiltration. In particular, distinction can be challenging from the immunohistochemical point of view in the case of high-grade mast cell disease which can be CD30 positive.

Keywords: Hodgkin's lymphoma, Systemic mastocytosis, Systemic mastocytosis with associated clonal haematological non-mast cell lineage disease (SM-AHNMD)

\footnotetext{
* Correspondence: vkloboves@onko-i.si

${ }^{4}$ Department of Cytology, Institute of Oncology, Zaloska 2, 1000 Ljubljana,

Slovenia

Full list of author information is available at the end of the article
}

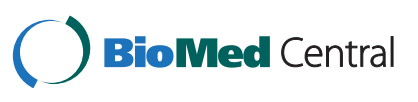

(c) 2015 Gasljevic et al.; licensee BioMed Central. This is an Open Access article distributed under the terms of the Creative Commons Attribution License (http://creativecommons.org/licenses/by/4.0), which permits unrestricted use, distribution, and reproduction in any medium, provided the original work is properly credited. The Creative Commons Public Domain Dedication waiver (http://creativecommons.org/publicdomain/zero/1.0/) applies to the data made available in this article, unless otherwise stated. 


\section{Background}

Mastocytosis is the result of a clonal, neoplastic proliferation of mast cells that accumulate in one or more organ systems. It is characterized by the presence of multifocal clusters or aggregates of abnormal mast cells. In the last WHO classification [1] of tumours of haematopoietic and lymphoid tissues, seven subtypes of mastocytosis are defined by the distribution of the disease and its clinical manifestations: cutaneous mastocytosis (CM), indolent systemic mastocytosis (ISM), systemic mastocytosis with associated clonal haematological non-mast cell lineage disease (SM-AHNMD), aggressive systemic mastocytosis (ASM), mast cell leukaemia (MCL), mast cell sarcoma (MCS) and extracutaneous mastocytoma. In CM, mast cell infiltrates are restricted to the skin, whereas systemic mastocytosis (SM) is characterised by involvement of at least one extracutaneous organ with or without involvement of the skin. Bone marrow is almost always involved in $\mathrm{SM}[1]$.

The association of systemic mastocytosis with a nonmast cell haematological neoplasm represents a specific subtype of mastocytosis termed SM-AHNMD [1]. It is the second most frequent variant of SM [2]. The overwhelming majority of the associated neoplasms are of myeloid origin, while lymphoid neoplasms associated with SM have been reported rarely [3-6]. Association of SM with Hodgkin's lymphoma has to be exceedingly rare; a review of the recent English literature on coexistence of Hodgkin's lymphoma and systemic mastocytosis resulted in only two published articles so far $[7,8]$. The objective of this study is to report the clinical and pathological features of a 37-year-old male patient who was diagnosed with Hodgkin's lymphoma of the upper mediastinum and indolent systemic mastocytosis which was found incidentally from bone marrow trephine biopsy performed for lymphoma staging.

\section{Case presentation}

A 37-year-old otherwise healthy male was referred to our institution because of a one-month lasting dysphagia of both hard and liquid food. He denied any breathing difficulties. There was no evidence of systemic symptoms. Physical examination showed tumour in the left jugular area measuring $2 \mathrm{~cm}$ in the largest diameter and some small, unsuspicious lymph nodes on both sides of the neck. The liver and spleen were not enlarged. There were no skin lesions. Peripheral blood counts were within normal limits. Serologies for anti-HAV, HbsAg, anti- HBc, anti-Hbs, anti-HCV and HIV were all negative. Lately performed laboratory studies revealed that the patient's serum tryptase level was $79 \mathrm{ng} / \mathrm{ml}$ (normal 2-10). Fine-needle aspiration biopsy (FNAB) of jugulare tumour was performed as part of the initial diagnostic procedure, but the sample obtained was not diagnostic.
Computer tomography of the thorax revealed a $5.2 \mathrm{~cm}$ large, hypodense, soft tissue tumour between the trachea and left arteria carotis communis, and subsequent ultrasound-guided FNAB was performed. The tumour was situated at the level of the upper thoracic opening. Cytologically, the diagnosis of a "neutrophil-rich" Hodgkin's lymphoma was established. Excisional biopsy of the tumour was performed to confirm the cytological findings. Bone marrow involvement with lymphoma was assessed by aspiration and trephine biopsy.

\section{Cytological and pathological findings}

The Giemsa-stained smear of the mediastinal tumour was very cellular. Neutrophils predominated, obscuring a few tumour cells found mainly along the edge of the smear. Tumour cells which were not degenerated showed moderately abundant, pale cytoplasm and single or multiple lobulated nuclei (Figure 1). Chromatin was finely granular. Nucleoli were conspicuous only in some cells. In a dense neutrophilic background, some histiocytes, few eosinophils, fibrin fibres and proteinaceous granular material were also observed. Because of the typical cytological picture and despite the fact that additional immunophenotypic analyses could not be performed (due to lack of tumour cells in the slides prepared for immunocytochemistry), the diagnosis of a "neutrophil-rich" Hodgkin's lymphoma was suggested.

Excisional biopsy of the mediastinal tumour revealed a lymphoid neoplasm composed of relatively rare mononuclear Hodgkin's and binuclear Reed-Sternberg cells residing in an infiltrate consisting of neutrophils, small lymphocytes, plasma cells, eosinophils and histiocytes (Figure 2). Some of the H-RS cells showed a retraction of the cytoplasmic membrane, creating the appearance of the so-called lacunar cells. Focally, HRS cells had more lobulated nuclei with smaller lobes. On the periphery of the

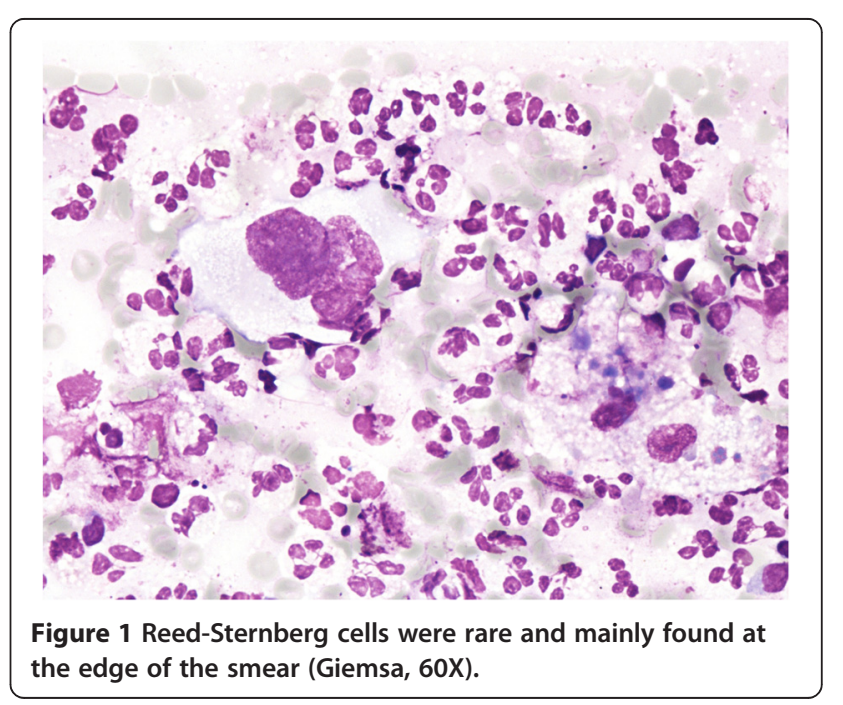




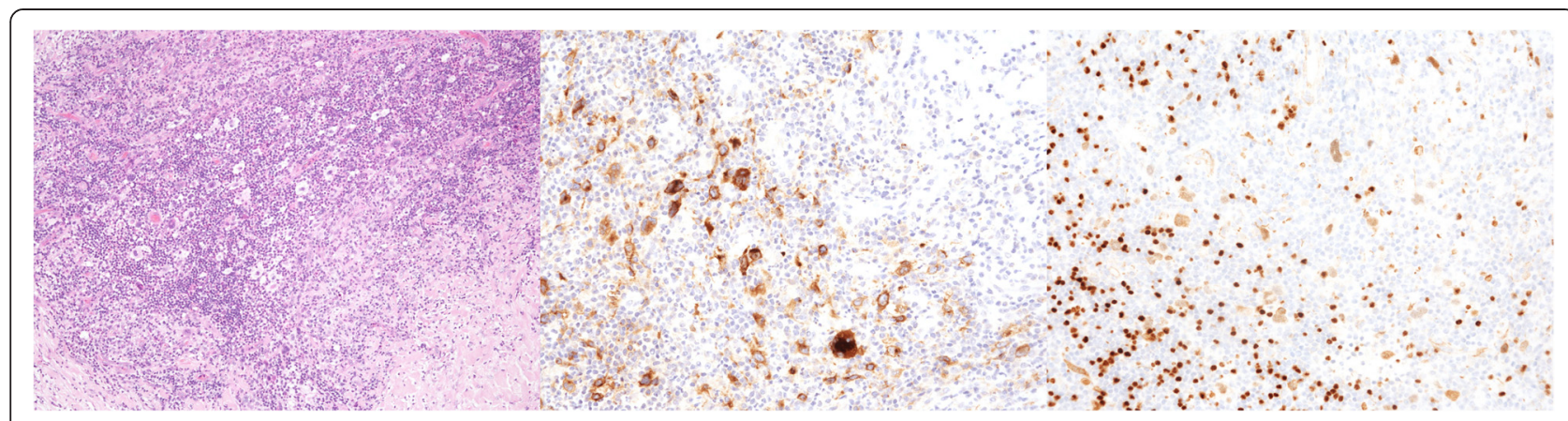

Figure 2 H\&E staining of mediastinal tumor, 10X; CD 30 and PAX 5 positive HRS cells, 20X.

specimen, a broad band of fibrosis was present. Large atypical cells were immunohistochemically positive for CD30, CD15, Pax5 and MUM1 (Figure 2). In situ hybridisation for EBV was negative. Based on the morphology of the H-RS cells, reactive infiltrate and present fibrosis, the tumour was classified as nodular sclerosis classical Hodgkin's lymphoma (NScHL).

Bone marrow trephine biopsy and the MGG-stained smear of the bone marrow aspirate performed for lymphoma staging revealed a slightly hypercellular bone marrow with some smaller clusters of atypical mast cells and a large peritrabecular infiltrate composed of atypical mastocytes and lymphocytes (Figure 3). Mast cells were highlighted by CD117 and tryptase immunostainings (Figure 4). CD25 and CD2 positivity of mast cells was consistent with their neoplastic phenotype (Figure 5). There were no HL infiltrates present in the bone marrow.

\section{Discussion}

According to the current WHO classification, patients with SM and concurrent haematological malignancy, either myeloid or lymphoid, are included in the group of so-called SM-AHNMD. Neoplasms associated with SM

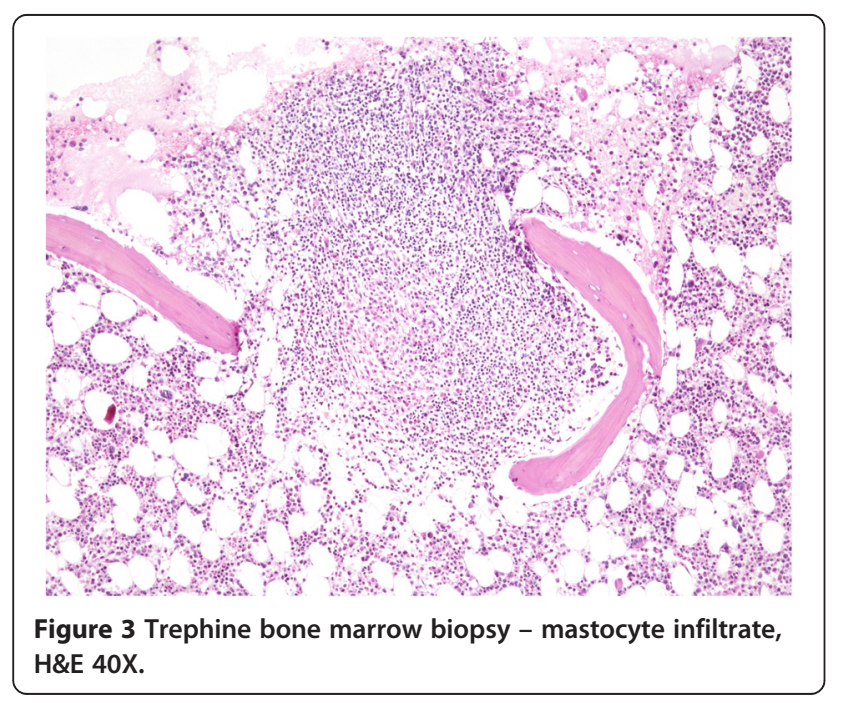

are mainly of myeloid origin, while lymphoproliferative diseases (LPD) are described only occasionally. It has been shown that in the case of SM associated with myeloid malignancies, the neoplastic mastocytes and malignant myeloid cells are clonally related, and both diseases are often diagnosed concurrently $[9,10]$. In contrast, patients with SM and LPD usually have a long history of indolent SM; LPD and SM clones are distinct [4]. The present case of SM-cHL is interesting in several aspects. First of all, the cytological picture of the mediastinal tumour was unusual because it mimicked suppurative lymphadenitis. Secondly, coexistence of HL and SM is exceedingly rare. To the best of the authors' knowledge, only two cases have been described in the English literature so far. Last but not least, patients with SM and LPD usually have a long history of indolent SM, which was not present in our patient.

From the cytological aspect, only a few cases of Hodgkin's lymphoma with numerous neutrophils have been described in the literature so far [11-14]. Histologically, these cases have been mostly classified as nodular sclerosis cHL, and only few of them as mixed cellularity cHL [11-14]. Such cases could be challenging because a predominance

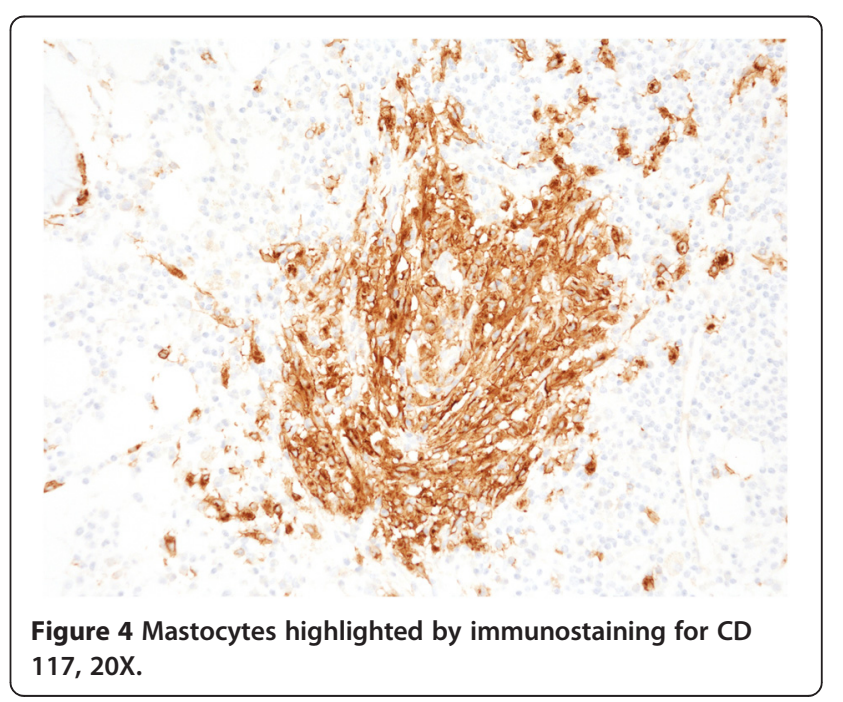




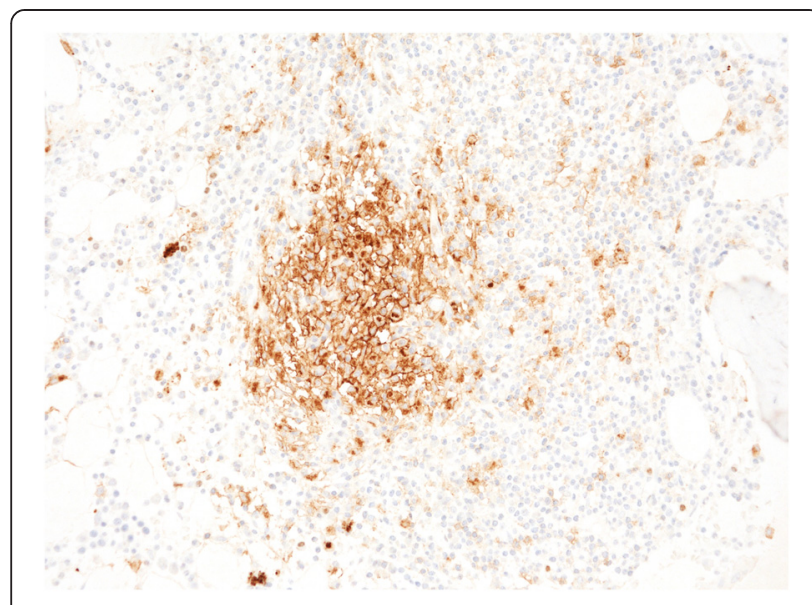

Figure 5 Abberant expression of CD25 on mastocyte cells, 60X.

of neutrophils and presence of necrosis can obscure the diagnostic Reed-Sternberg and Hodgkin cells, and they can be misdiagnosed as suppurative lymphadenitis [11-14]. To avoid a false-negative cytological diagnosis, an accurate search for Reed-Sternberg and Hodgkin cells must be carry out. In the differential diagnosis of "neutrophil-rich" HL and suppurative lymphadenitis, "neutrophil-rich" anaplastic large T-cell lymphoma (ALCL) and metastatic carcinoma must be considered. Suppurative lymphadenitis is most commonly found in tuberculosis, Calmette Guerin bacillus and atypical mycobacterial infection, Kikuchi's disease, cat-scratch disease, and lymphogranuloma venereum $[13,14]$. Identification of the infectious agent is crucial for the diagnosis, therefore a part of the FNAB sample must be sent for microbiological testing. "Neutrophil-rich" ALCL may be difficult to differentiate from "neutrophilrich" Hodgkin's lymphoma. Immunocytochemical staining for CD30, CD15, ALK, CD45, CD20, EMA and T-cell markers can resolve this differential diagnostic dilemma $[15,16]$. Metastatic carcinoma could also represent a differential diagnostic possibility. Metastatic squamous-cell carcinoma is particularly prone to necrosis and inflammation, which in many cases leads to the cytological picture that can be very similar to that in "neutrophil-rich" HL or ALCL [14]. However, this dilemma could be easily solved by cytokeratin immunocytochemical staining.

Coexistence of SM with HL might be challenging also in terms of separation of bone marrow infiltrates. Reactive lymphoid aggregates are a feature of bone marrow infiltrates in patients with indolent SM. They can be composed of a mixture of mast cells and lymphocytes, or either of them may predominate [17]. As well, increased eosinophils can accompany lymphocytes as a part of the background infiltrate not only in systemic mastocytosis but also in HL. Since HL is also characterised by a paucity of neoplastic cells in comparison to background reactive cells, morphological similarity between the infiltrates of
SM and HL could cause confusion; either of them can be misdiagnosed. Furthermore, aggressive mastocytosis (ASM) is morphologically characterised by a marked degree of bone marrow infiltration that can be diffuse or focal. Its major differential diagnosis is SM-AHNMD since AHNMD may be almost obscured by the massive mast cell infiltrate [18]. Besides, it has been shown that a major proportion of cases with high-grade SM (ASM, SM-AHNMD and mast cell leukaemia) show a strong expression of CD30 by a majority of neoplastic mast cells [18]. All of the abovementioned, as well as CD30 positivity of mast cells, may result in the misinterpretation of bone marrow infiltration by HL, unless antitryptase staining is performed, and may contribute to erroneous tumour staging and treatment.

\section{Conclusions}

In conclusion, we report a very rare combination of systemic mastocytosis with Hodgkin's lymphoma as associated clonal haematological non-mast cell lineage disease. In our case, HL was presented as a mediastinal tumour and sub-classified as nodular sclerosis. Systemic mastocytosis was an unexpected finding. To our knowledge, this is the third reported case in the literature of such disease combination.

\section{Consent}

Written informed consent was obtained from the patient for publication of this Case Report and any accompanying images. A copy of the written consent is available for review by the Editor-in-Chief of this journal.

\section{Abbreviations \\ CM: Cutaneous mastocytosis; ISM: Indolent systemic mastocytosis; \\ SM-AHNMD: Systemic mastocytosis with associated clonal haematological non-mast cell lineage disease; ASM: Aggressive systemic mastocytosis; MCL: Mast cell leukaemia; MCS: Mast cell sarcoma; FNAB: Fine-needle aspiration biopsy; NSCHL: Nodular sclerosis classical Hodgkin's lymphoma; MGG: May Grinwald Giemsa; LPD: Lymphoproliferative diseases; \\ ALCL: Anaplastic large T-cell lymphoma; CHL: Classical Hodgkin's lymphoma.}

\section{Competing interests}

The authors declare that they have no competing interests.

\section{Authors' contribution}

GG and VKP: Have made substantial contributions to conception and design, acquisition of data, analysis and interpretation of data; have written the manuscript and have been involved in drafting it and revising it critically for important intellectual content. KGB, AG, MS and BG: Have been involved in drafting the manuscript or revising it critically for important intellectual content. All authors have given final approval of the version to be published.

\section{Author details}

'Department of Pathology, Institute of Oncology, Zaloska 2, 1000 Ljubljana, Slovenia. ${ }^{2}$ Department of Laboratory medicine, Institute of Oncology, Zaloska 2, 1000 Ljubljana, Slovenia. ${ }^{3}$ Department of Hematology, University Medical Centre Ljubljana, 1000 Ljubljana, Slovenia. ${ }^{4}$ Department of Cytology, Institute of Oncology, Zaloska 2, 1000 Ljubljana, Slovenia.

Received: 31 October 2014 Accepted: 25 February 2015

Published online: 14 March 2015 


\section{References}

1. Horny HP, Metcalfe DD, Bennet JM, Bain BJ, Akin C, Escribano L, et al. Systemic mastocytosis. In: Swerdlow S, Campo E, Lee Harris N, Jaffe ES, Pileri SA, Stein H, Thiele J, Vardiman JW, editors. WHO Classification of Tumours of Haematopoietic and Lymphoid Tissue. Lyon: IARC; 2008. p. 54-63.

2. Horny HP, Sotlar K, Sperr WR, Valent P. Systemic mastocytosis with associated clonal haematological non-mast cell lineage diseases: a histopathological challenge. J Clin Pathol. 2004;57:604-8.

3. Horny HP, Sotlar K, Stellmacher F, Valent P, Grabbe J. An unusual case of systemic mastocytosis associated with chronic lymphocytic leukaemia (SM-CLL). J Clin Pathol. 2006;59:264-8.

4. Kim Y, Weiss LM, Chen YY, Pullarkat V. Distinct clonal origins of systemic mastocytosis and associated B-cell lymphoma. Leuk Res. 2007;31:1749-54.

5. Fernandez-Torres R, Verea MM, Alvarez A, Torres P, Fonseca E. Systemic mastocytosis associated with splenic marginal zone lymphoma with villous lymphocytes. Derm Res Pract. 2011. doi:10.1155/2011/385074.

6. Travis WD, Li CY, Bergstralh EJ, Yam LT, Swee RG. Systemic mast cell disease. Analysis of 58 cases and literature review. Medicine. 1988;67:345-68.

7. Cox JV, Balaban EP, Demian SE, Sheehan RG. An eosinophilic fibrohistiocytic lesion of bone marrow in a patient with Hodgkin's disease. A potential for morphologic confusion. Cancer. 1991;68:1824-7.

8. Saletti P, Ghielmini M, Scali G, Pedrinis E, Gueneau M, Cavalli F. Hodgkin's and Castelman's diseases in a patient with systemic mastocytosis. Ann Hematol. 1999;78:97-100.

9. Pullarkat VA, Bueso-Ramos C, Lai R, Kroft S, Wilson CS, Pullarkat ST, et al. Systemic mastocytosis with associated clonal hematological non-mast cell lineage disease: analysis of clinicopathologic features and activating c-kit mutations. Am J Hematol. 2003;73:12-7.

10. Pullarkat V, Bedell V, Kim Y, Bhatia R, Nakamura R, Forman S, et al. Neoplastic mast cells in systemic mastocytosis associated with $t(8 ; 21)$ acute myeloid leukemia are derived from the leukemic clone. Leuk Res. 2007;31:261-5.

11. Lopes Cardozo P. Atlas of clinical cytology. London: Heinemann; 1976. p. $110-5$.

12. Fulciniti F, Zeppa P, Vetrani A, Troncone G, Palombini L. Hodgkin's disease mimicking suppurative lymphadenitis: a possible pitfall in fine-needle aspiration cytology. Diagn Cytopathol. 1989;5:282-5.

13. Tani E, Ersöz C, Svedmyr E, Skoog L. Fine-needle aspiration cytology and immunocytochemistry of Hodgkin's disease suppurative type. Diagn Cytopathol. 1998;18:437-40.

14. Vicandi B, Jiménez-Heffernan JA, López-Ferrer P, Gamallo C, Viguer JM. Hodgkin's disease mimicking suppurative lymphadenitis: a fine-needle aspiration report of five cases. Diagn Cytopathol. 1999;20:302-6.

15. Jhala DN, Medeiros LJ, Lopez-Terrada D, Jhala NC, Krishnan B, Shahab I. Neutrophil-rich anaplastic large cell lymphoma of T-cell lineage. Am J Clin Pathol. 2000;114:478-82.

16. Creager AJ, Geisinger KR, Bergman S. Neutrophil rich Ki-1-positive anaplastic large cell lymphoma. Am J Clin Pathol. 2002;117:709-15.

17. Horny HP, Kaiserling E. Lymphoid cells and tissue mast cells of bone marrow lesions in systemic mastocytosis: a histological and immunohistological study. Br J Hematol. 1988;69:449-55.

18. Horny HP, Sotlar K, Valent P. Differential diagnosis of systemic mastocytosis in routinely processed bone marrow biopsy specimens: a review. Pathobiology. 2010;77:169-80.

\section{Submit your next manuscript to BioMed Central and take full advantage of:}

- Convenient online submission

- Thorough peer review

- No space constraints or color figure charges

- Immediate publication on acceptance

- Inclusion in PubMed, CAS, Scopus and Google Scholar

- Research which is freely available for redistribution 